\title{
Gastrostomy Tube for Nutrition and Malignant Bowel Obstruction in Patients With Cancer
}

Gabrielle Gauvin, MD¹; Chi Chi Do-Nguyen, BS ${ }^{1}$; Johanna Lou, MD1; Eileen Anne O'Halloran, MD'; Leigh T. Selesner, MD; Elizabeth Handorf, $\mathrm{PhD}^{1}$; Molly E. Collins, MD ${ }^{1}$; and Jeffrey M. Farma, MD ${ }^{1}$

\begin{abstract}
Background: Gastrostomy tubes (G-tubes) are invaluable clinical tools that play a role in palliation and nutrition in patients with cancer. This study aimed to better understand the risks and benefits associated with the placement and maintenance of G-tubes. Methods: Patients who underwent placement of a G-tube for cancer from January 2013 through December 2017 at a tertiary care center were considered for inclusion. Clinical data were retrospectively collected from medical records. Results: A total of 242 patients with cancer, whose average age at diagnosis was 61 years (range, 21-94 years), underwent G-tube placement for nutrition (76.4\%), decompression $(22.7 \%)$, or both $(0.8 \%)$. Successful insertion was achieved in $96.8 \%$, but 8 patients required $>1$ attempted method of insertion. In the decompression group, minor postplacement complications were less common ( $23.6 \%$ vs $53.5 \% ; P<.001)$ and survival was shorter $(P<.001)$ compared with the nutrition group. For those with decompressive G-tubes, $45.5 \%$ had a palliative care consult; $56.4 \%$ were seen by social workers; and $46.3 \%$ went to hospice. The frequency of hospice discharge was higher in patients who had consults $(53.7 \%$ vs $23.1 \%$; $P=.01$ ). Conclusions: Half of the patients who received decompressive G-tubes presented with stage IV disease and died within 1 month of placement. Those with $>1$ consult were more likely to be discharged to hospice. Patients with G-tubes for nutrition saw no change in functionality, complication rate, or survival, regardless of adjunct chemotherapy status. These findings illustrate the need for a tool to allow a better multidisciplinary approach and interventional decision-making for patients with cancer.
\end{abstract}

J Natl Compr Canc Netw 2021;19(1):48-56 doi: 10.6004/jnccn.2020.7604

\footnotetext{
1Department of Surgical Oncology, Fox Chase Cancer Center, Temple University Health System, Philadelphia, Pennsylvania.
}

\section{Background}

Gastrostomy tubes (G-tubes) are invaluable clinical tools that have an important role in palliation for patients with cancer diagnoses. The most common indication is for long-term enteral nutrition in patients unable to maintain sufficient oral intake. The prevalence of malnutrition in patients with cancer has been reported to range from $20 \%$ to $70 \% .^{1}$ G-tubes have also been used for gastric decompression in patients with malignant bowel obstruction (MBO). The global prevalence of MBO is estimated to be between $3 \%$ and $15 \%$ for patients with cancer, reaching $20 \%$ to $50 \%$ among patients with ovarian cancer and $10 \%$ to $20 \%$ among patients with colorectal cancer. ${ }^{2,3}$

Despite the frequent use of G-tubes for palliation, high-quality data studying their efficacy and associated clinical outcomes in this patient population are limited. The current literature is predominantly composed of studies performed in gynecologic or head and neck cancer populations. Moreover, many of these investigations focus on percutaneous endoscopic gastrostomy (PEG) tubes with little mention of interventional radiology (IR)-guided and surgically placed G-tubes. The few studies performed in mixed populations of patients with cancer and PEG tubes have shown short-term survival to be high (up to $90 \%$ at 1 month) and long-term survival to be low (as low as $37.7 \%$ at $12-18$ months and $20 \%$ at 3 years)..$^{4-9}$ It was also shown that patient populations with PEG tubes sustained significantly less reduction in nutritional parameters. ${ }^{10,11}$

Furthermore, limited data exist on the efficacy of PEG tubes in alleviating symptoms of MBO. One retrospective review found a complete resolution of symptoms in $91 \%$ of patients with decompressive PEG tubes for colorectal, pancreatic, or gynecologic cancer. ${ }^{8,12}$ These data are crucial because many patients with advanced or incurable gastrointestinal malignancies have decreased survival rates, and G-tubes continue to be placed in truly palliative situations with few randomized data to support their benefit. Additionally, recent studies have shown that patients who receive an early palliative 
care consultation as part of their treatment live longer with better quality of life, despite their reception of less cancer-directed therapy. ${ }^{13}$ However, past literature reports a wide range between $27.5 \%$ and $76.9 \%$ of patients who had access to palliative care services before death due to advanced malignancy. ${ }^{14,15}$ The aim of this study was to better understand the risks, benefits, and practices associated with the placement and maintenance of palliative G-tubes in patients with cancer at our institution.

\section{Methods}

\section{Data Collection}

After obtaining approval from the Fox Chase Cancer Center Institutional Review Board, we identified and retrospectively reviewed the clinical charts of 257 patients who were considered for or had undergone placement of a G-tube at an NCI-designated Cancer Center from January 2013 through December 2017. We excluded 6 patients due to placement of non-G-tubes (jejunostomy, cecostomy + ileostomy, or colostomy tube), 8 patients due to failure of insertion of a G-tube placement, and 1 patient due to the absence of a cancer diagnosis. A retrospective chart review was performed for the remaining 242 patients with a primary cancer diagnosis and successful G-tube placement.

Clinical data were collected from electronic medical records, including demographics, indication and technique for G-tube placement, and preprocedure and postprocedure clinical variables. Pathologic data included TNM classification and clinical stage as defined by the AJCC. Indications for G-tube placement were collected from physician records. The indication for nutrition was defined as dysphagia secondary to radiation, surgery, or worsening tumor burden; decreased per os tolerance secondary to chemotherapy or worsening tumor burden; concern for aspiration; or malnutrition at the time of G-tube placement. The indication for decompression of MBO was defined as the presence of gastric outlet obstruction, gastrointestinal dysmotility, nonoperable small bowel obstruction, or nonoperable large bowel obstruction at the time of G-tube placement. The indication for both nutrition and decompression was defined as having $>1$ indication for nutrition and $>1$ indication for decompression at the time of G-tube placement. Clinical outcomes included postoperative complications during admission and after discharge, status at last follow-up, and date of death. Based on prior work by Grant et al, ${ }^{16}$ postoperative complications were stratified into major and minor complications. Major complications were defined as pneumoperitoneum, perigastric hematoma, aspiration pneumonia, or fatality caused by the insertion procedure, whereas minor complications were defined as minor G-tube site infection or G-tube malfunction.

\section{Statistical Analysis}

Patient demographics and clinical characteristics were summarized using counts and percentages for categorical variables and with means and standard deviations for continuous variables. Differences in covariates between groups of interest were assessed using the chisquare, Fisher exact, $t$, or Wilcoxon test, as appropriate. Changes in measures such as weight and albumin concentrations were assessed using paired $t$ tests. Survival was calculated from the date of G-tube placement to the date of death or last follow-up visit, with patients alive at last follow-up considered censored. Survival curves were calculated using the Kaplan-Meier method, and differences between curves were assessed using logrank tests. Median follow-up time was determined using the reverse Kaplan-Meier method. $P$ values $<.05$ were considered statistically significant, and all tests were 2 -sided. Multiple testing was not accounted for. Statistical analyses were conducted using STATA, version 15 (StataCorp LLC).

\section{Results}

A total of 242 patients with cancer with an average age at diagnosis of 63.4 years (range, 35-94 years) underwent G-tube placement for nutrition (76.4\%), decompression for MBO $(22.7 \%)$, or both $(0.8 \%)$ (Table 1). Successful insertion was achieved in $96.7 \%$ of patients, but 8 patients required $>1$ method of insertion attempt. The 2 patients with G-tube placement for both nutrition and decompression were removed from subsequent analysis because of the added complexity of statistical analysis. These 2 patients constituted $0.8 \%$ of the patient population, and a sensitivity analysis was deemed not necessary and thus was not conducted. In the nutrition group, the method of insertion was $88.1 \%$ in IR, $8.1 \%$ in the operating room, and $3.8 \%$ in the endoscopy suite (ES), compared with $81.8 \%, 14.5 \%$, and $3.6 \%$, respectively, in the MBO group $(P=.36)$. Active treatment within 3 months of G-tube insertion was seen in $38.3 \%$ of the nutrition group versus $29.1 \%$ in the $\mathrm{MBO}$ group $(P=.22)$. Overall, major postplacement complications were similar between the 2 groups, but minor postplacement complications were significantly less common in the MBO group than in the nutrition group $(20.0 \%$ vs $50.3 \%$; $P<.001)$. Median follow-up time was 40.1 months. Survival after G-tube placement was significantly shorter in the $\mathrm{MBO}$ group than in the nutrition group $(P<.001)$, with $54.8 \%$ versus $90.7 \%$ survival at 30 days, $11.4 \%$ versus $60.0 \%$ survival at 1 year, and $9.5 \%$ versus $41.1 \%$ survival at 3 years. 


\section{Table 1. Patient Demographics}

\begin{tabular}{|c|c|c|c|c|}
\hline & $\begin{array}{l}\text { Overall } \\
\text { n (\%) }\end{array}$ & $\begin{array}{c}\text { Nutrition } \\
\text { n (\%) }\end{array}$ & $\begin{array}{c}\text { Decompression } \\
n(\%)\end{array}$ & P Value ${ }^{a}$ \\
\hline \multicolumn{5}{|l|}{ Patient characteristics } \\
\hline Number of patients & $240(100)$ & $185(76.4)$ & $55(22.7)$ & \\
\hline Mean age at diagnosis \pm SD (range), $y$ & $63.4 \pm 11.2(35-94)$ & $64.6 \pm 11.0(36-94)$ & $59.5 \pm 11.3(35-88)$ & .003 \\
\hline ASA score at placement, mean \pm SD $(n=176)$ & $2.46 \pm 0.57$ & $2.43 \pm 0.55$ & $2.58 \pm 0.66$ & .2 \\
\hline \multicolumn{5}{|l|}{ Tumor characteristics } \\
\hline Type of primary malignancy & & & & $<.001$ \\
\hline Gl, pancreas, or liver & $32(13.3)$ & $8(4.3)$ & $24(43.6)$ & \\
\hline Head and neck & $138(57.0)$ & $138(74.6)$ & $0(0.0)$ & \\
\hline Thoracic/Esophageal & $37(15.4)$ & $34(18.4)$ & $3(5.5)$ & \\
\hline Gynecologic/Genitourinary & $30(12.5)$ & $4(2.2)$ & $26(47.3)$ & \\
\hline Other & $3(2.5)$ & $1(0.5)$ & $2(3.6)$ & \\
\hline Stage $(n=194)$ & & & & .53 \\
\hline I & $7(3.6)$ & $5(3.5)$ & $2(4.1)$ & \\
\hline II & $24(12.4)$ & $18(12.4)$ & $6(12.2)$ & \\
\hline III & $38(19.6)$ & $25(17.2)$ & $13(26.5)$ & \\
\hline IV & $125(49.2)$ & $97(66.9)$ & $28(57.1)$ & \\
\hline \multicolumn{5}{|l|}{ Treatment } \\
\hline Ascites drained before placement & & & & $<.001$ \\
\hline Present and drained & $11(4.6)$ & $0(0.0)$ & $11(20.0)$ & \\
\hline Present and undrained & $24(10.0)$ & $2(1.1)$ & $22(40.0)$ & \\
\hline No ascites & $205(85.4)$ & $183(98.9)$ & $20(20.0)$ & \\
\hline Chemotherapy within 3 mo of placement $(n=238)$ & & & & .22 \\
\hline Yes & $86(35.9)$ & $70(38.3)$ & $16(29.1)$ & \\
\hline No & $152(63.2)$ & $113(61.7)$ & $39(70.9)$ & \\
\hline \multicolumn{5}{|l|}{ Consultations before placement } \\
\hline Palliative and hospice $(n=239)$ & $52(21.8)$ & $27(14.7)$ & $25(45.5)$ & $<.001$ \\
\hline Speech & $123(51.3)$ & $123(66.5)$ & $0(0.0)$ & $<.001$ \\
\hline Swallow & $75(31.3)$ & $75(40.5)$ & $0(0.0)$ & $<.001$ \\
\hline Nutrition & $101(42.1)$ & $94(50.8)$ & $7(12.7)$ & $<.001$ \\
\hline Social work & $83(34.6)$ & $52(28.1)$ & $31(56.4)$ & $<.001$ \\
\hline G-tube placement method & & & & .36 \\
\hline IR & $208(86.7)$ & $163(88.1)$ & $45(81.8)$ & \\
\hline Operating room & $23(9.6)$ & $15(8.1)$ & $8(14.5)$ & \\
\hline Endoscopy suite & $9(3.8)$ & $7(3.8)$ & $2(3.6)$ & \\
\hline Required TPN postplacement & $47(19.6)$ & $16(8.7)$ & $31(56.4)$ & $<.001$ \\
\hline \multicolumn{5}{|l|}{ Outcomes } \\
\hline Complications & & & & .001 \\
\hline None & $128(53.3)$ & $86(46.5)$ & $42(76.4)$ & \\
\hline Major & $8(3.3)$ & $6(3.2)$ & $2(3.6)$ & \\
\hline Minor & $104(43.3)$ & $92(50.3)$ & $11(20.0)$ & \\
\hline \multicolumn{5}{|l|}{ Pain score, mean (SD) $(n=176)$} \\
\hline Before placement & $3.0(3.0)$ & $2.9(3.3)$ & $3.6(3.6)$ & .25 \\
\hline 1 mo after placement (change) & $-1.9(3.9)$ & $-2.0(3.8)$ & $-1.4(4.4)$ & .51 \\
\hline
\end{tabular}




\begin{tabular}{|c|c|c|c|c|}
\hline Discharge disposition & & & & $<.001$ \\
\hline Home & $183(76.6)$ & $161(87.0)$ & $22(40.7)$ & \\
\hline Hospice & $32(13.4)$ & $7(3.8)$ & $25(46.3)$ & \\
\hline Survival, \%a & & & & $<.001$ \\
\hline $30 d$ & 82.7 & 90.7 & 54.8 & \\
\hline $1 \mathrm{y}$ & 49.1 & 60.0 & 11.4 & \\
\hline $3 y$ & 33.7 & 41.1 & 9.5 & \\
\hline
\end{tabular}

Abbreviations: ASA, American Society of Anesthesiologists physical status; GI, gastrointestinal; G-tube, gastrostomy tube; IR, interventional radiology; SNF, skilled nursing facility; TPN, total parenteral nutrition.

aBold indicates statistically significant $P$ value.

bPercent survival from Kaplan-Meier curve.

\section{Nutrition}

A total of 185 patients underwent G-tube placement for nutritional support, with an average age at placement of 64.6 years (range, $36-94$ years). There was no difference between the $37.5 \%$ of patients receiving active chemotherapy and those who were not in terms of tumor stage at placement, placement technique, or patient American Society of Anesthesiologists physical status score (Table 2). There was no difference in postprocedure mortality between the 2 groups. Patients undergoing active treatment had a minor complication rate of $51.4 \%$ versus $49.6 \%$ in those not undergoing treatment $(P=.426)$.

Despite successful G-tube insertion, $8.2 \%$ of patients still required total parenteral nutrition (TPN). One month after G-tube placement, the mean weight of both groups had decreased from preprocedure weight $(2.8 \mathrm{~kg}$ in treatment group vs $1.9 \mathrm{~kg}$ in nontreatment group; $P=$.189). Overall, among patients who underwent G-tube placement for nutritional support, the mean weight decreased by 2.3 kilograms 1 month after placement $(P<.001)$. Serum albumin and prealbumin concentrations were recorded before G-tube insertion and 1 month postprocedure. Before G-tube insertion, patients in the treatment group had higher albumin concentrations than those in the nontreatment group (3.18 vs $2.88 \mathrm{~g} / \mathrm{dL} ; P=.003$ ). However, no significant posttreatment difference between the groups was noted (Table 2), and, overall, there was no significant change in serum albumin and prealbumin concentrations 1 month after G-tube placement $(P=.48$ and $P=.69$, respectively). Overall, pain scores decreased by 1.4 points $(P<.001)$. The 30 -day, 1 -year, and 3 -year survival rates were not significantly different between those undergoing active treatment and those not undergoing active treatment.

\section{Decompression}

A total of 55 patients underwent decompressive G-tube placement, with an average age at placement of 59.5 years (range, 35-88 years) (Table 3). Overall mortality in this group was $90.6 \%$. However, only $45.5 \%$ of patients had a palliative care consult, $56.4 \%$ were seen by a social worker, $25.5 \%$ had both consults, and $23.6 \%$ had neither consult. In the group that had a palliative care consult, a social work consult, or both $(n=42)$, placement was achieved in IR for $78.6 \%$ of patients, in ES for $2.4 \%$, and in the operating room for $19.1 \%$. The group without a consult showed similar rates, at $92.3 \%, 7.7 \%$, and $0 \%$, respectively $(P=.13)$. Complications occurred in $23.6 \%$ of the cohort, with a rate of $21.4 \%$ in those with a consult versus $30.8 \%$ in those without $(P=.56)$. Overall, $46.3 \%$ of the patients were discharged to hospice. The frequency of hospice discharge among patients who had consults was higher than among those who did not $(53.7 \%$ vs $23.1 \%$; $P=.01$ ) (Figure 1). Of the patients with MBO discharged to hospice, $88.0 \%$ had either a palliative care and/or social work consult, and $36.0 \%$ had both a palliative care and a social work consult. There was no difference in survival between the 2 groups: 30-day and 1-year survival were $50.2 \%$ and $12.6 \%$, respectively, among patients with a consult compared with $69.2 \%$ and $7.7 \%$, respectively, among patients without a consult (Figure 2).

\section{Discussion}

Although prior studies limited their patient populations to either gynecologic or head and neck malignancies, this study is one of the few to include a mixed population of patients with cancer, allowing better generalizability. More than half of the patients had head and neck tumors, but there was also a large proportion of esophageal, gastrointestinal, pancreatic, hepatic, and gynecologic/genitourinary cancers. This is in line with 


\section{Table 2. G-Tube Placement for Nutritional Support Stratified by Chemotherapy Status}

\begin{tabular}{|c|c|c|c|c|}
\hline & $\begin{array}{l}\text { Overall } \\
\text { n (\%) }\end{array}$ & $\begin{array}{c}\text { Active Treatment } \\
n(\%)\end{array}$ & $\begin{array}{c}\text { No Active Treatment } \\
n(\%)\end{array}$ & P Value ${ }^{a}$ \\
\hline \multicolumn{5}{|l|}{ Patient characteristics } \\
\hline Number of patients & $183(100)$ & $70(38.3)$ & $113(61.8)$ & \\
\hline Mean age at diagnosis (range), $y$ & $64.6(36-94)$ & $61.8(36-90)$ & $66.4(44-94)$ & .01 \\
\hline Mean ASA score at placement \pm SD $(n=141)$ & $2.43 \pm 0.55$ & $2.34 \pm 0.52$ & $2.50 \pm 0.57$ & .1 \\
\hline \multicolumn{5}{|l|}{ Tumor characteristics } \\
\hline Type of primary malignancy, n (\%) & & & & .25 \\
\hline Gl, pancreas, or liver & $8(4.3)$ & $5(7.1)$ & $3(2.7)$ & \\
\hline Head and neck/thoracic & $170(92.9)$ & $65(92.9)$ & $105(92.9)$ & \\
\hline Gynecologic/Genitourinary & $4(2.2)$ & $0(0.0)$ & $4(3.5)$ & \\
\hline Other & $1(0.6)$ & $0(0.0)$ & $1(0.9)$ & \\
\hline Stage $(n=144)$ & & & & .58 \\
\hline I & $5(3.5)$ & $1(1.6)$ & $4(4.9)$ & \\
\hline II & $18(12.5)$ & $8(12.9)$ & $10(12.2)$ & \\
\hline III & $25(17.4)$ & $13(21.0)$ & $12(14.6)$ & \\
\hline IV & $96(66.7)$ & $40(64.5)$ & $56(66.3)$ & \\
\hline \multicolumn{5}{|l|}{ Treatment characteristics } \\
\hline G-tube insertion method & & & & .99 \\
\hline IR & $161(88.0)$ & $61(87.1)$ & $100(88.5)$ & \\
\hline Operating room & $15(8.2)$ & $6(8.6)$ & $9(8.0)$ & \\
\hline Endoscopy suite & $7(3.8)$ & $3(4.3)$ & $4(3.5)$ & \\
\hline Required TPN postplacement & $15(8.2)$ & $6(8.6)$ & $9(8.0)$ & .99 \\
\hline \multicolumn{5}{|l|}{ Outcomes } \\
\hline Mean weight loss after $1 \mathrm{mo}(\mathrm{SD}), \mathrm{kg}$ & $2.3(9.4)$ & $2.8(8.9)$ & $1.9(9.8)$ & .189 \\
\hline \multicolumn{5}{|l|}{ Mean serum albumin \pm SD } \\
\hline Before G-tube & $3.00 \pm 0.74$ & $3.18 \pm 0.82$ & $2.88 \pm 0.68$ & .003 \\
\hline 1 mo after G-tube & $3.26 \pm 0.72$ & $3.33 \pm 0.65$ & $3.19 \pm 0.77$ & .48 \\
\hline \multicolumn{5}{|l|}{ Mean serum prealbumin \pm SD } \\
\hline Before G-tube & $12.11 \pm 4.84$ & $12.34 \pm 4.22$ & $11.90 \pm 5.40$ & .63 \\
\hline 1 mo after G-tube & $11.16 \pm 4.31$ & $11.00 \pm 3.78$ & $11.32 \pm 5.14$ & .69 \\
\hline Complications & & & & .30 \\
\hline None & $85(46.5)$ & $30(42.9)$ & $55(48.7)$ & \\
\hline Major & $6(3.2)$ & $4(5.7)$ & $2(1.8)$ & \\
\hline Minor & $92(50.3)$ & $36(51.4)$ & $56(49.6)$ & \\
\hline Survival, \%b & & & & .16 \\
\hline $1 \mathrm{y}$ & 75.9 & 69.6 & 80.0 & \\
\hline $3 y$ & 52.5 & 48.8 & 55.2 & \\
\hline
\end{tabular}

Abbreviations: ASA, American Society of Anesthesiologists physical status; G-tube, gastrostomy tube; Gl, gastrointestinal; IR, interventional radiology; TPN, total parenteral nutrition.

aBold indicates statistically significant $P$ value.

bPercent survival from Kaplan-Meier curve, patients surviving $>3$ mo.

what would be expected, given that common indications for G-tube placement in cancer populations are long-term enteral nutrition and gastric decompression of MBO.

Regarding the technique of G-tube placement, $86.7 \%$ of patients had G-tubes placed under radiologic guidance by IR. The bulk of literature is limited to PEG tubes, and the use of radiologic guidance for G-tube placement can range from $2 \%$ to $37.4 \% .^{8,17}$ Brooksbank et $\mathrm{al}^{8}$ studied G-tube placement for malignant intestinal obstruction and reported $90.2 \%$ of G-tubes were placed endoscopically, $7.8 \%$ were placed surgically, and only $2.0 \%$ were placed under radiologic guidance. Our study 


\begin{tabular}{|c|c|c|c|c|}
\hline & $\begin{array}{c}\text { Overall } \\
\text { n (\%) }\end{array}$ & $\begin{array}{c}\text { Consult } \\
\text { n (\%) }\end{array}$ & $\begin{array}{c}\text { No Consult } \\
\text { n (\%) }\end{array}$ & $P$ Value $^{a}$ \\
\hline \multicolumn{5}{|l|}{ Patient characteristics } \\
\hline Number of patients & $55(100)$ & $42(76.4)$ & $13(23.6)$ & \\
\hline Type of primary malignancy, n (\%) & & & & .05 \\
\hline Gl, pancreas, or liver & $24(43.6)$ & $14(33.3)$ & $10(76.9)$ & \\
\hline Head and neck/thoracic & $3(5.5)$ & $3(7.1)$ & $0(0.0)$ & \\
\hline Gynecologic/Genitourinary & $26(47.3)$ & $23(54.8)$ & $3(23.1)$ & \\
\hline ॥ & $6(12.2)$ & $4(10.8)$ & $2(16.7)$ & \\
\hline III & $13(26.5)$ & $10(27.0)$ & $3(25.0)$ & \\
\hline IV & $28(57.1)$ & $21(56.8)$ & $7(58.3)$ & \\
\hline \multicolumn{5}{|l|}{ Treatment } \\
\hline Chemotherapy within 3 mo of placement & $16(29.1)$ & $10(23.8)$ & $6(46.2)$ & .16 \\
\hline \multicolumn{5}{|l|}{ Consult pattern } \\
\hline Palliative care & $25(45.5)$ & $25(59.5)$ & $0(0.0)$ & \\
\hline Social work & $31(56.4)$ & $31(73.8)$ & $0(0.0)$ & \\
\hline G-tube insertion method & & & & .13 \\
\hline Complications & & & & .56 \\
\hline None & $42(76.4)$ & $33(78.6)$ & $9(69.2)$ & \\
\hline Major & $2(3.6)$ & $1(2.4)$ & $1(7.7)$ & \\
\hline Minor & $11(20.0)$ & $8(19.0)$ & $3(23.1)$ & \\
\hline Discharge to hospice & $25(46.3)$ & $22(53.7)$ & $3(23.1)$ & .01 \\
\hline Survival ${ }^{b}$ & & & & .48 \\
\hline $30 d$ & $54.8 \%$ & $50.2 \%$ & $69.2 \%$ & \\
\hline $1 \mathrm{y}$ & $11.4 \%$ & $12.6 \%$ & $7.7 \%$ & \\
\hline $3 y$ & $9.5 \%$ & $10.0 \%$ & $\mathrm{~N} / \mathrm{A}$ & \\
\hline
\end{tabular}

Abbreviations: G-tube, gastrostomy tube; GI, gastrointestinal; IR, interventional radiology; N/A, not applicable.

aBold indicates statistically significant $P$ value.

bPercent survival from Kaplan-Meier curve.

reported a higher use of radiologic guidance $(87 \%)$ than in other studies, and the findings are comparable to those of a retrospective review by Shaw et al, ${ }^{18}$ who investigated palliative decompression G-tubes placed by IR and found a $72 \%$ success rate on the first attempt. Similarly, Wollman and D'Agostino ${ }^{17}$ found that the success rate for tube placement was higher for percutaneous radiologic gastrostomy than for PEG (100\% vs $95 \%)$, and percutaneous radiologic gastrostomy was subsequently performed in $66.7 \%$ of patients in whom PEG placement failed.

Our study also addressed outcomes that impact patients' quality of life. Mean pain scores decreased overall. Although nearly half of patients experienced 
A
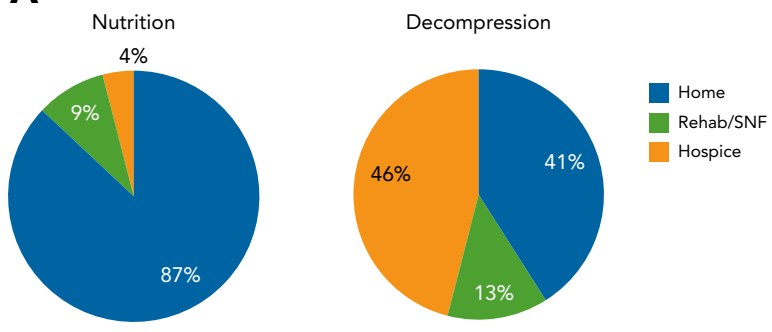

B
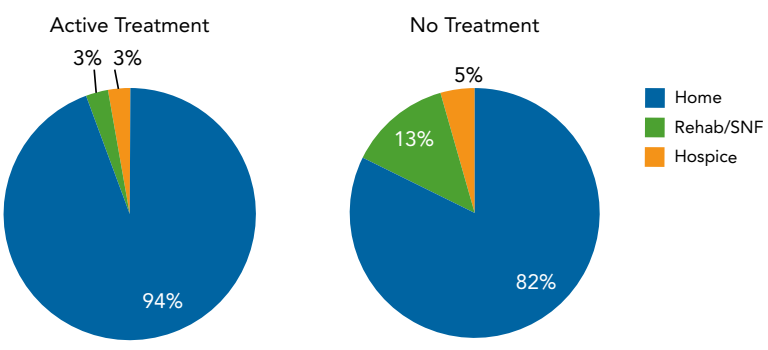

C
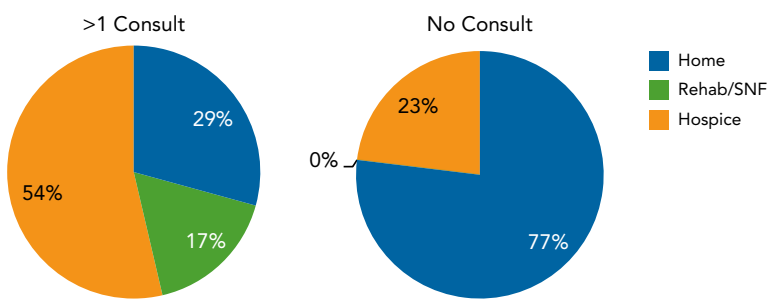

Figure 1. Discharge patterns of patients receiving a gastrostomy tube, comparing (A) nutrition versus decompression, (B) active treatment versus no treatment with nutrition tubes, and $(C)$ consult versus no consult for decompression tubes.

Abbreviations: rehab, rehabilitation facility; SNF, skilled nursing facility.

postplacement complications, only $3.3 \%$ were considered major complications, which is lower than the currently reported range of $4.3 \%$ to $19.1 \%$ of major complications and $40.0 \%$ of minor complications, advocating the use of IR insertion. ${ }^{12,16}$ Three-fourths of all patients were discharged to home after their G-tube placement.
Furthermore, the survival rates of patients are comparable to those seen in previous studies, with short-term survival rates at 30 days higher than long-term survival rates at 1 and 3 years. This suggests that G-tube insertion by IR may offer a more minimally invasive option with potentially equivalent survival compared with PEG tube placement. Although this study did not break down survival by placement method, this is an area for exploration in future studies.

Although the risks and benefits of G-tube placement are well-known, our goal was to stratify these risks and benefits in terms of indication for G-tube placement. Furthermore, with the use of radiologic guidance for G-tube placement in $86.7 \%$ of patients, the risks and benefits described will add to the literature on the implications of this minimally invasive option.

\section{Nutrition}

Most of the patients received G-tube placement for nutritional support. TPN independence or advancement of diet failed for only a small percentage of patients. Nutritional and functional status, using prealbumin and albumin concentrations and weight as surrogate measures, were not drastically affected by the placement of a G-tube. Overall, the mean weight decreased by 2.3 kilograms 1 month after placement $(P<.001)$,but there was no significant change in prealbumin and albumin concentrations. A prospective study by Corry et $\mathrm{al}^{11}$ investigated the effect of PEG tubes versus nasogastric tubes on the nutritional outcomes in patients with head and neck cancer undergoing chemoradiation, and found that patients with PEG tube placement sustained significantly less weight loss at 6 weeks after treatment (median, $0.8 \mathrm{~kg}$ gain vs $3.7 \mathrm{~kg}$ loss; $P<.001$ ). Another prospective study by Sadasivan et $\mathrm{al}^{10}$ found similar results; however, patients with IR and surgically placed G-tubes, in addition to PEG tubes, were included in the study.

Patients with G-tubes for nutritional support experienced significantly more minor complications than those with decompressive G-tubes. This could be
A

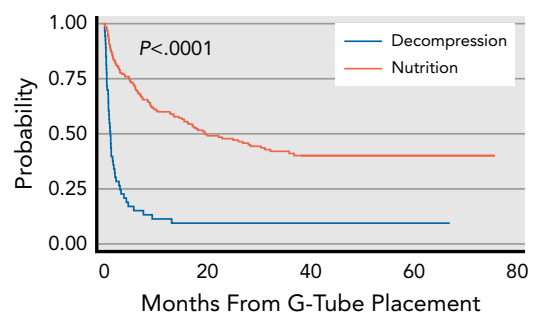

B

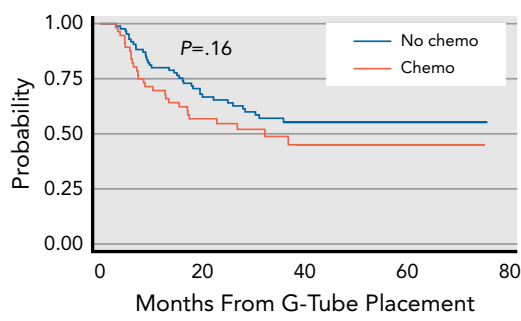

C

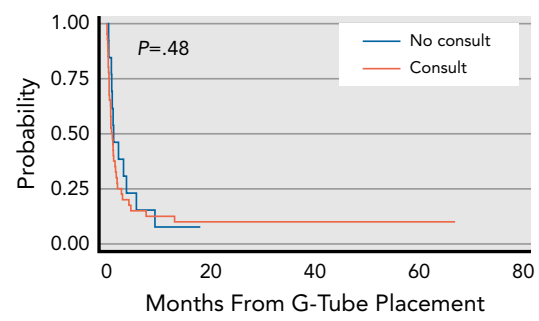

Figure 2. Kaplan-Meier curves for overall survival comparing (A) nutrition versus decompression, (B) active treatment versus no treatment with nutrition tubes, and $(\mathrm{C})$ consult versus no consult for decompression tubes.

Abbreviations: chemo, chemotherapy; G-tube, gastrostomy tube. 
attributed to the better survival rate in the nutritional support group. A retrospective study by Nguyen et $\mathrm{al}^{19}$ examined PEG tube placement in patients receiving chemoradiation for head and neck cancer and reported a $3 \%$ complication rate and $0 \%$ mortality rate, concluding that G-tube feeding is safe in this population. Although most patients did not receive chemotherapy within 3 months of G-tube placement, the results indicated similar nutritional status, complication rates, and mortality rates between those who underwent active treatment and those who did not. This finding suggests that placement of G-tubes for nutritional support is safe, with minimal effect on functional status, which is an important finding, because many medical oncologists are hesitant to perform procedural interventions in patients receiving active cytotoxic chemotherapy.

\section{Decompression}

In evaluating the role of G-tubes for palliation of obstructive symptoms, we found that most patients in this study were not receiving active treatment. The role of decompressive G-tubes for MBO have been studied previously by Brooksbank et $\mathrm{al}^{8}$ and Issaka et $\mathrm{al}^{12}$ who both supported the consideration of G-tubes in providing patients with symptomatic relief. Life expectancy after G-tube placement is poor in patients requiring decompression for $\mathrm{MBO}$ compared with those requiring nutritional support, with $>50 \%$ surviving $<1$ month. Perhaps due to the low life expectancy in advanced malignancy, minor postplacement complications were significantly less common in the MBO group than in the nutrition group. There is a high rate of successful G-tube placement and a high rate of relief of obstructive symptoms after G-tube placement, suggesting an important role for G-tubes in comfort care for these patients.

In addition, $40 \%$ of these patients had ascites present and undrained during their G-tube insertion. A review of the literature by Shaw et $\mathrm{al}^{18}$ noted that many previous studies did not address management of ascites in patients with G-tubes for MBO, and those that did were composed primarily of patients with pre-G-tube ascites drainage. Future studies can be conducted to investigate the impact of undrained ascites on G-tube placement.

Despite the significantly shorter survival of patients with a decompressive G-tube, only three-fourths received a palliative care or social work consult before tube insertion. Patients who received a palliative care consult, social work consult, or both were more likely to be discharged to hospice than patients who did not receive a consult. Sedhom and Barile ${ }^{14}$ and de Oliveira Valentino et $\mathrm{al}^{15}$ found that patients with gynecologic malignancies had significantly greater referral to palliative care services, but patients with upper gastrointestinal tract cancer were associated with late palliative care consults (odds ratio, 2.42; $P=.001$ ). If patients are referred to palliative care, the overall aggressiveness of care in the last year of life is significantly less, and the patients receive less chemotherapy in the last 2 weeks of life. More important, Sedhom and Barile ${ }^{14}$ found that patients who were not referred to palliative care services experienced death more frequently in the intensive care unit $(16 \%$ vs $2 \% ; P<.001)$ and the emergency department ( $12 \%$ vs $1 \%$; $P<.001)$ compared with patients who were referred to palliative care services. With only $45.5 \%$ of patients receiving a palliative care consult and $56.4 \%$ receiving a social work consult, there is much room for improvement at our institution in involving multidisciplinary teams. Providers must be well versed in palliative management and recognize the importance of early palliative care referral to optimize the individual's quality of life and care in these complex clinical, emotional, spiritual, social, and existential situations. ${ }^{13}$ In future studies, we plan to create a tool or checklist to provide an organized multidisciplinary approach to G-tube placement and care for patients with advanced malignancy to ensure early palliative care referral at our institution.

\section{Limitations}

This study was conducted at a tertiary referral cancer center. Nearly half of the patients presented with stage IV disease. With a 30 -day survival rate of $54.8 \%$, a substantial percentage of the patients in the decompression group were not included in long-term results. This potentially affects the outcomes of the analysis and makes comparison between the nutrition and decompression groups difficult.

Prospective studies are needed to reveal factors affecting the decision process and patient selection for placement of G-tubes. If these results are validated in other population-based studies, these predictors of complications and survival after referral for G-tube placement could be used to identify patients with a low probability of survival who may not benefit from receiving a G-tube.

\section{Conclusions}

G-tubes play an important palliative role in patients with advanced malignancies who need nutritional support or decompression of MBO. Because of their longer survival, patients with G-tubes for nutrition had more minor complications. Regardless of adjunct chemotherapy status, patients with nutritional G-tubes saw minimal change in functionality, with prealbumin and albumin concentrations and weight as surrogate measures; complication rate; or short- and long-term survival. Nearly half of patients who received decompressive G-tubes presented with stage IV disease and died within 
1 month of placement. Those with at least one consult were more likely to be discharged to hospice.

Submitted March 14, 2020; accepted for publication June 10, 2020.

Author contributions: Study concept, design: Gauvin, Collins, Farma. Data extraction: Do-Nguyen, Lou, Selesner. Statistical analysis: O'Halloran, Handorf.
Manuscript preparation: Do-Nguyen, Lou, Selesner. Critical revision: Do-Nguyen, Lou, O'Halloran, Selesner, Handorf.

Disclosures: The authors have disclosed that they have not received any financial consideration from any person or organization to support the preparation, analysis, results, or discussion of this article.

Correspondence: Gabrielle Gauvin, MD, 40116 Lionel-Emond Street, Gatineau, Qc J8Y 1WZ, Canada. Email: ggauvinmd@gmail.com

\section{References}

1. Keung EZ, Liu X, Nuzhad A, et al. In-hospital and long-term outcomes after percutaneous endoscopic gastrostomy in patients with malignancy. J Am Coll Surg 2012;215:777-786.

2. Ripamonti $C$, Mercadante S. Pathophysiology and management of malignant bowel obstruction. In: Hanks GWC, Cherny NI, Christakis NA, et al eds. Oxford Textbook of Palliative Medicine, 4th ed. Oxford, UK: Oxford University Press; 2010:850-862.

3. Tuca A, Guell E, Martinez-Losada E, et al. Malignant bowel obstruction in advanced cancer patients: epidemiology, management, and factors influencing spontaneous resolution. Cancer Manag Res 2012;4:159-169.

4. Taylor CA, Larson DE, Ballard DJ, et al. Predictors of outcome after percutaneous endoscopic gastrostomy: a community-based study. Mayo Clin Proc 1992;67:1042-1049.

5. Finocchiaro C, Galletti R, Rovera G, et al. Percutaneous endoscopic gastrostomy: a long-term follow-up. Nutrition 1997;13:520-523.

6. Grant MD, Rudberg MA, Brody JA. Gastrostomy placement and mortality among hospitalized Medicare beneficiaries. JAMA 1998;279:1973-1976.

7. Kobayashi K, Cooper GS, Chak A, et al. A prospective evaluation of outcome in patients referred for PEG placement. Gastrointest Endosc 2002;55:500-506

8. Brooksbank MA, Game PA, Ashby MA. Palliative venting gastrostomy in malignant intestinal obstruction. Palliat Med 2002;16:520-526.

9. Sofue K, Takeuchi Y, Tsurusaki M, et al. Value of percutaneous radiologic gastrostomy for patients with advanced esophageal cancer. Ann Surg Oncol 2016;23:3623-3631.

10. Sadasivan A, Faizal B, Kumar M. Nasogastric and percutaneous endoscopic gastrostomy tube use in advanced head and neck cancer patients: a comparative study. J Pain Palliat Care Pharmacother 2012; 26:226-232.

11. Corry J, Poon W, McPhee N, et al. Prospective study of percutaneous endoscopic gastrostomy tubes versus nasogastric tubes for enteral feeding in patients with head and neck cancer undergoing (chemo)radiation. Head Neck 2009;31:867-876.

12. Issaka RB, Shapiro DM, Parikh ND, et al. Palliative venting percutaneous endoscopic gastrostomy tube is safe and effective in patients with malignant obstruction. Surg Endosc 2014;28:1668-1673.

13. Lambert LA, Wiseman J. Palliative management of peritoneal metastases. Ann Surg Oncol 2018;25:2165-2171.

14. Sedhom R, Barile D. Palliative care referrals and related outcomes among advanced cancer patients: a study revealing our institutional shortcomings [abstract]. J Clin Oncol 2017;35(Suppl):Abstract e21506.

15. de Oliveira Valentino TC, Paiva BSR, de Oliveira MA, et al. Factors associated with palliative care referral among patients with advanced cancers: a retrospective analysis of a large Brazilian cohort. Support Care Cancer 2018;26:1933-1941.

16. Grant DG, Bradley PT, Pothier DD, et al. Complications following gastrostomy tube insertion in patients with head and neck cancer: a prospective multi-institution study, systematic review and meta-analysis. Clin Otolaryngol 2009;34:103-112.

17. Wollman B, D'Agostino HB. Percutaneous radiologic and endoscopic gastrostomy: a 3-year institutional analysis of procedure performance. AJR Am J Roentgenol 1997;169:1551-1553.

18. Shaw $C$, Bassett RL, Fox PS, et al. Palliative venting gastrostomy in patients with malignant bowel obstruction and ascites. Ann Surg Oncol 2013;20: 497-505.

19. Nguyen NP, North D, Smith HJ, et al. Safety and effectiveness of prophylactic gastrostomy tubes for head and neck cancer patients undergoing chemoradiation. Surg Oncol 2006;15:199-203. 